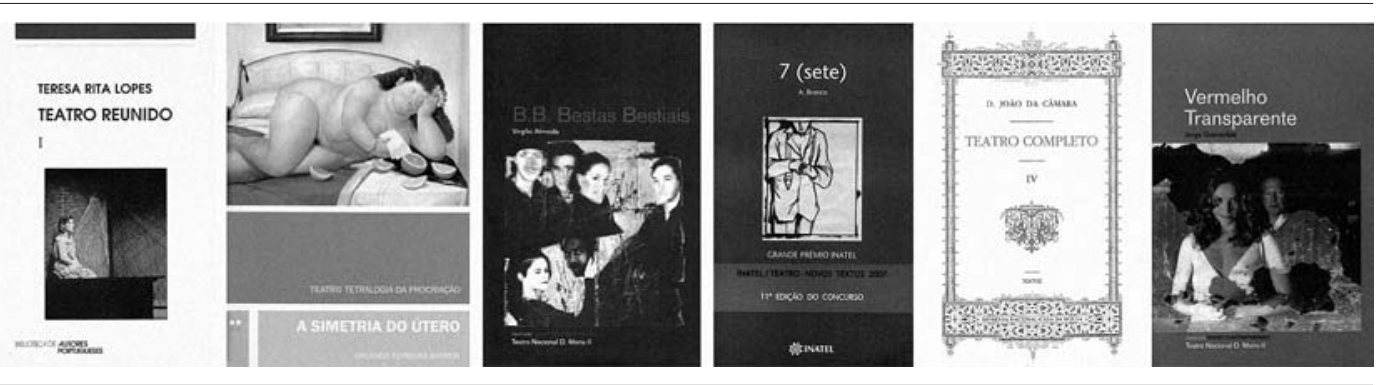

\title{
Publicações de teatro em 2007
}

\section{Lista compilada por Sebastiana Fadda}

\section{Peças originais (ou volumes de peças) em primeira edição}

A., RUBEN, Triálogo; Júlia; Relato 1453, ed. Liberto Cruz, pref. Armando Nascimento Rosa, Lisboa, Assírio \&t Alvim, 2007.

AA.W., Autos, passos e bailinhos: Os textos dos Bonecos de Santo Aleixo, coord. Christine Zurbach, José Alberto Ferreira e Paula Seixas, Évora, Casa do Sul / Centro Dramático de Évora (CENDREV) / Centro de História da Arte e Investigação Artística da Universidade de Évora, 2007, 315 pp.

AA.W., Panos: Palcos novos, palavras novas (Auto do Branco de Neve, de Armando Silva Carvalho; Copo meio vazio, de Alexandre Andrade; Justamente, de Ali Smith, trad. Miguel Castro Caldas), Lisboa, Culturgest, 2007.

AA.W., Teatro português do século XVI, vol. I, Teatro profano, tomo I (Auto do caseiro de Alvalade, Auto dos escrivães do pelourinho, Auto do escudeiro surdo, Auto de Florisbel, Auto de Guiomar do Porto), ed. e introd. José Camões, Lisboa, Imprensa Nacional-Casa da Moeda, 2007.

AA.W.Teatro romântico português: 0 drama histórico, prefácio, selecção e notas de Luiz Francisco Rebello, Lisboa, Imprensa-Nacional-Casa da Moeda, 2007, 413 pp.

ALMEIDA, Virgílio, B.B. Bestas bestiais, Lisboa, Teatro Nacional D. Maria II, 2007.

BARROS, Orlando Ferreira, A elegia da madre seca, Viana do Castelo, Centro Cultural do Alto Minho, 2007.

--, A simetria do útero, Viana do Castelo, Centro Cultural do Alto Minho, 2007.

BORGES, Paulo, Folia: Mistério de uma noite de Pentecostes, Lisboa, Ésquilo, 2007.

BRANCO, A., 7 (sete), Lisboa, Grande Prémio INATEL do Concurso INATEL/Teatro - Novos Textos, 2007.

CÂMARA, D. João da, et al. [Gervásio Lobato, Henrique Lopes de Mendonça, Eduardo Schwalbach, Moura Cabral, Jaime Batalha Reis, Fernando Caldeira e Delfim Guimarães], Teatro completo, vol. IV: Obras em colaboração (Zé Palonso, 0 burro do sr. alcaide, 0 burro em pancas, 0 solar dos barrigas, Cocô, Reineta e Facada, 0 testamento da velha, Bibi \&t C. ${ }^{a}$, Aldeia na corte,

O João das Velhas), org. e notas Rita Martins, Lisboa, Imprensa Nacional-Casa da Moeda, 2007.

COSTA, Artur, Camilo e Ana Augusta, Porto, Campo das Letras, 2007.
COSTA, Mário da, Monólogo de um actor com o seu público, Porto, Papiro Editora, 2007.

ESCOBAR, Carlos Henrique, Teatro (Médeia masculina (José Medéia); A tragédia de Althusser (Paixão do Marxismo); Ana Clitemnestra; A caixa de cimento), Águeda, Moïra, 2007.

GUIMARÃES, Jorge, Vermelho transparente, Lisboa, Teatro Nacional D. Maria II, 2007.

LOPES, Teresa Rita, Teatro reunido, org. e pref. Sebastiana Fadda, Lisboa, Imprensa Nacional-Casa da Moeda, 2 vols., 2007.

MELO, Jorge Silva, Fala da criada nos Noialles que no fim de contas vamos descobrir chamar-se também Séverine numa noite do Inverno de 1975 em Hyères, Lisboa, Livros Cotovia, 2007.

MENDES, José Maria Vieira, A minha mulher; Onde vamos morar, Lisboa, Artistas Unidos / Livros Cotovia, Livrinhos de Teatro, 2007.

MORGADO, Paulo, O corrupto e o diabo, Lisboa, D. Quixote, 2007. MESOUITA, Marcelino, Teatro completo, vol. II (O Regente, 0 tirano da bela Urraca, 0 sonho da Índia, Peraltas e sécias, O auto do busto, A morta galante, Sempre noiva), org. Duarte Ivo Cruz, Lisboa, Imprensa Nacional-Casa da Moeda, 2007.

NEGREIROS, João, Os vendilhões do templo; Silêncio, introd. José Manuel Mendes, s/l, Teatro Universitário do Minho, 2007.

OLIVEIRA, Mickael de, O queé teu entregou aos mortais, Lisboa, Maria Matos, 2007

PATRíCIO, António, O fim (história dramática em dois quadros); Diálogo na Alhambra, ed. Armando Nascimento Rosa, Lisboa, Assírio \&t Alvim, 2007.

PESSOA, Carlos J., Comédia em 3 actos, Lisboa, Teatro da Garagem, 2007.

--, Teatro-clip, Lisboa, Teatro da Garagem, 2007.

--, A morte de Danton na garagem, Lisboa, Teatro da Garagem, 2007.

POPPE, Manuel, Pedro I, Lisboa, Editorial Teorema, 2007.

PORTELA, Patrícia, Odilia ou a história das musas confusas do cérebro de Patrícia Portela, Lisboa, Editorial Caminho, 2007. TAVARES, Rui, O arquitecto, Lisboa, Edições Tinta-da-china, 2007. TORRADO, António, A casa da lenha, posfácio do autor, Porto, Campo das Letras, 2007. 

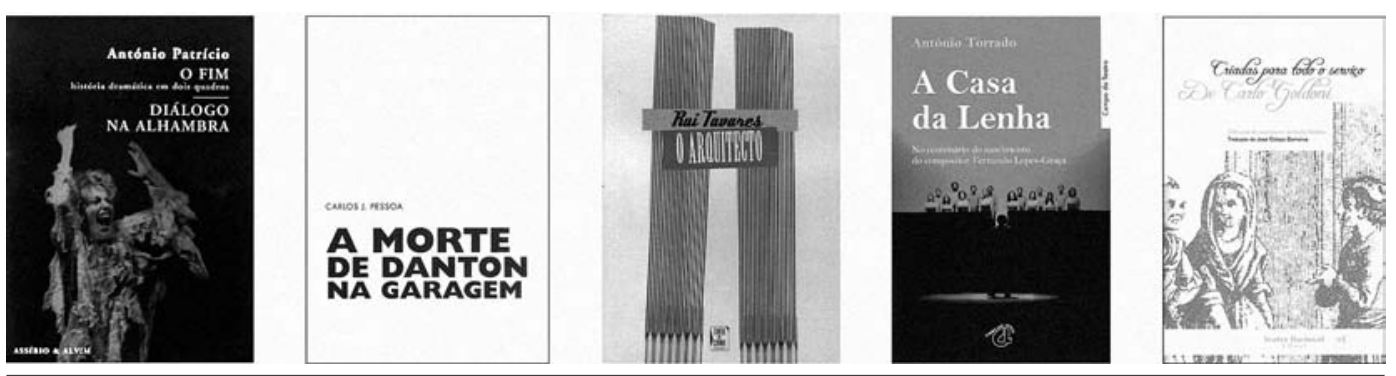

\section{Peças em reedição}

BRANDÃO, Raul / PASCOAES, Teixeira de, Jesus Cristo em Lisboa, Lisboa, Assírio \&t Alvim, 2007.

\section{Traduções}

BENET I JORNET, Josep, Desejo e outras peças (E.R.; Precisamente hoje; 0 quarto do miúdo), trad. Ângelo Ferreira de Sousa e Joana Frazão, Lisboa, Artistas Unidos / Livros Cotovia, Livrinhos de Teatro, 2007.

BRECHT, Bertolt, Histórias do senhor Keuner. Versão de Zurique, trad. Maria Hermínia Brandão, Porto, Campo das Letras, Campo do Teatro, 2007.

GOLDONI, Carlo, Criadas para todo o serviço, trad. José Colaço Barreiros, Lisboa, Teatro Nacional D. Maria II, 2007.

JOSEPHSON, Erland, Uma peça de teatro, trad. Solveig Nordlund e Joana Frazão, introd. Solveig Nordlund, s/l, Livros de Areia, Teatro, 2007.

KAUFMAN, Moisés, O projecto Laramie, trad. Felipa Mourato, Lisboa, Maria Matos, 2007.

MAALOUF, Amin, Adriana Mater, trad. António Pescada, Lisboa, Difel, 2007.

MAYORGA, Juan, Hamelin, trad. António Gonçalves, Lisboa, Artistas Unidos / Livros Cotovia, Livrinhos de Teatro, 2007.

MACDONAGH, Martin, The Pillowman:O homem almofada, trad. Tiago Guedes, Lisboa, Maria Matos, 2007.

MOLIĖRE, O misantropo, edição bilingue, trad. e pref. Vasco Graça Moura, Lisboa, Bertrand Editora, 2007.

NERUDA, Pablo, Fulgor e morte de Joaquín Murieta, trad. e pref. José Viale Moutinho, Porto, Campo das Letras, 2007.

O' CASEY, Sean, A charrua e as estrelas, trad. Helena Barbas, s/l, Livros de Areia, Teatro, 2007.

ORTON, Joe, O saque, trad. Luísa Costa Gomes, Porto, Campo das Letras, 2007.

PASOLINI, Pier Paolo, Calderón, trad. Mário Feliciano e António Barahona, Lisboa, Artistas Unidos / Livros Cotovia, Livrinhos de Teatro, 2007.

--, Pillades, trad. Mário Feliciano e Luiza Neto Jorge, Lisboa, Artistas Unidos / Livros Cotovia, Livrinhos de Teatro, 2007.

ROSTAND, Edmond, Cirano de Bergerac, edição bilingue, trad. Vasco Graça Moura, Lisboa, Livraria Bertrand, 2007.

SCHWAB, Werner, As presidentes; PESO A MAIS, sem peso: SEM FORMA, trad. Vera San Payo de Lemos, Lisboa, Artistas Unidos / Livros Cotovia, Livrinhos de Teatro, 2007.

SHANLEY, John Patrick, Dúvida: Uma parábola, trad. Felipa Mourato e Ana Luisa Guimarães, Lisboa, Maria Matos, 2007.

SHAKESPEARE, William, Canseiras de amor em vão, trad., introd. e notas de Rui Carvalho Homem, Porto, Campo das Letras, 2007.

--, Júlio César, trad. José Manuel Mendes, Luis Lima Barreto e Luís Miguel Cintra, Lisboa, Livros Cotovia, 2007.

--, Romeu e Julieta: A mais bela história de amor de todos os tempos, trad. Fernando Villas-Boas, il. João Fazenda, Cruz Quebrada, Oficina do Livro, 2007.

SÓFOCLES, Antígona, introd., versão e notas de Maria Helena da Rocha Pereira, Lisboa, Fundação Calouste Gulbenkian, (7a ed. rev.), 2007.

--, Filoctetes, introd., versão e notas José Ribeiro Ferreira, Lisboa, Fundação Calouste Gulbenkian (4. ${ }^{a}$ ed.), 2007.

TARANTINO, Antonio, Vésperas da Virgem Santíssima; Brilharetes, trad. Tereza Bento, Lisboa, Artistas Unidos / Livros Cotovia, Livrinhos de Teatro, 2007.

TCHÉKHOV, Anton, 0 cerejal: Comédia em quatro actos, trad. António Pescada, Porto, Campo das Letras, Colecção Teatro Nacional S. João, 2007. 

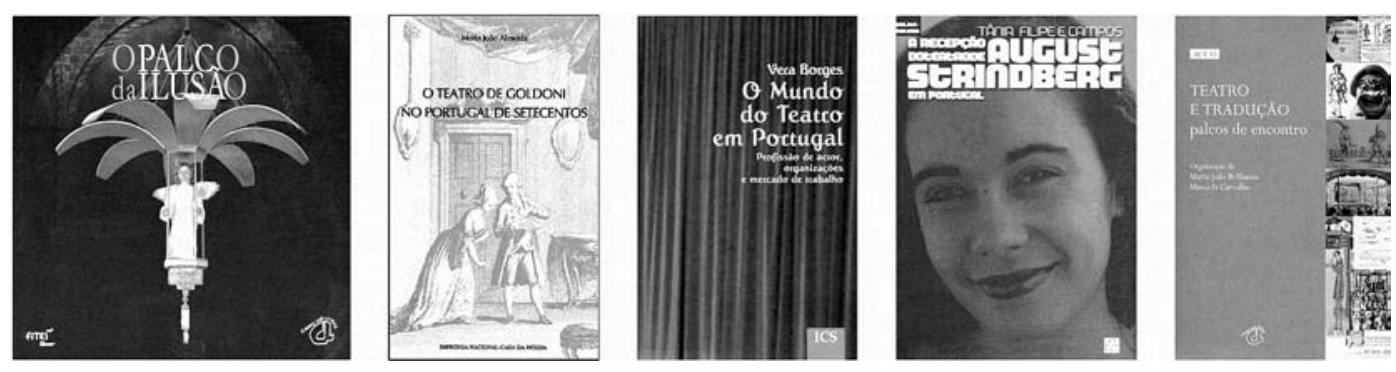

\section{Estudos / Documentos}

AA.W., O palco da ilusão, catálogo da exposição apresentada no âmbito do XXX FITEl, Porto, Campo das Letras / Antiqua Escenas, 2007.

AA.W., Um teatro sem teatro, catálogo da exposição, Lisboa, Museu Colecção Berardo, Arte Moderna e Contemporânea / Museu d'Art Contemporani de Barcelona, 2007.

ALMEIDA, Maria João, 0 teatro de Goldoni no Portugal de Setecentos, Lisboa, Imprensa Nacional-Casa da Moeda, 2007.

ALVAREZ, José Carlos (coord.), Rostos e poses no teatro português ou a Irrepetivel presença próxima de algo sempre muito distante, Lisboa, Museu Nacional do Teatro, 2007.

BORGES, Vera, 0 mundo do teatro em Portugal: Profissão de actor, organizações e mercado de trabalho, Lisboa, Imprensa de Ciências Sociais, 2007.

BRILHANTE, Maria João / CARVALHO, Manuela (org.), Teatro e tradução: Palcos de encontro, ACT 15, Porto, Campo das Letras, 2007.

CAMPOS, Tânia Filipe e, A recepção do teatro de August Strindberg em Portugal, Évora, Caleidoscópio, 2007.

DOMINGOS, Nuno, A Ópera do Trindade: 0 papel da Companhia Portuguesa de Ópera na "educação cultural" do Estado Novo, Lisboa, ASA / Lua de Papel Série Músicas, Vol. 1, 2007.

FRANÇA, José Augusto, 0 essencial sobre António Pedro, Lisboa, Imprensa Nacional-Casa da Moeda, 2007.

GARCIA, Orlando et al., Formações e profissões nas artes e ofícios do espectáculo: Artes cénicas e performativas em Portugal 2006-2007, Lisboa, Chapitô, 2007.

GOLDBERG, RoseLee, A arte da performance: Do futurismo ao presente, trad. Jefferson Luiz Camargo, adapt. e rev. Carla Oliveira e Rui Lopes, Lisboa, Orpheu Negro, 2007.

GOUVEIA, Marcelo (coord.) / BRILHANTE, Maria João (comissariado científico), 0 que é o teatro?, Lisboa, Direcção Geral das Artes, Território Artes, 2007.

MARTINS, Rita, Raul Brandão do texto à cena, Lisboa, Imprensa Nacional-Casa da Moeda, 2007.

MATOS, Luis de, Manual de caracterização, Lisboa, INATEL, 2007.

MELO, Jorge Silva, Século passado, Lisboa, Livros Cotovia, 2007.

MENDES, Conceição, Manual de produção cultural: Algumas reflexões sobre o tema, Lisboa, INATEL, 2007.
PACHECO, Natércia / CALDAS, José / TERRASÊCA, Manuela (org.), Teatro e educação: Transgressões transdisciplinares, Porto, Edições Afrontamento, 2007.

RIBEIRO, João Mendes, Arquitecturas em palco / Architectures on Stage, Coimbra, Instituto das Artes / Ministério da Cultura / Almedina, 2007.

ROSA, Armando Nascimento, Peças breves no teatro escrito de Natália Correia, Separata da Revista Forma Breve, n. ${ }^{\circ}$, Aveiro, Universidade de Aveiro / Centro de Linguas e Culturas, 2007.

SANTOS, Ana Clara / VASCONCELOS, Ana Isabel, Repertório teatral na Lisboa oitocentista (1835-1846), Lisboa, Imprensa Nacional-Casa da Moeda, 2007.

SILVA, Maria de Fátima Sousa e, Ensaios sobre Aristófanes, Lisboa, Cotovia, 2007.

TRIGO, Jorge / REIS, Luciano, Chaby Pinheiro: Um senhor actor (1873-1933), Lisboa, SeteCaminhos, 2007.

ZURBACH, Christine, A tradução teatral: O texto e a cena, Évora, Caleidoscópio, 2007.

\section{Publicações periódicas}

Adágio, n. ${ }^{\circ}$ 42, 1. ${ }^{\circ}$ Semestre de 2007, dir. Mário Barradas, Évora, Centro Dramático de Évora (CENDREV).

Artistas Unidos: Revista, n. 19 (Julho 2007) e n. 20 (Dezembro 2007), dir. Jorge Silva Melo, Lisboa, Livros Cotovia.

Obscena: Revista de artes performativas, n. 5 (JunhoJulho 2007), Lisboa, dir. Tiago Bartolomeu Costa.

Sinais de cena, nºs. 7 (Junho 2007) e 8 (Dezembro 2007), dir. Maria Helena Serôdio, Associação Portuguesa de Críticos de Teatro \& Centro de Estudos de Teatro, Porto, Campo das Letras. 

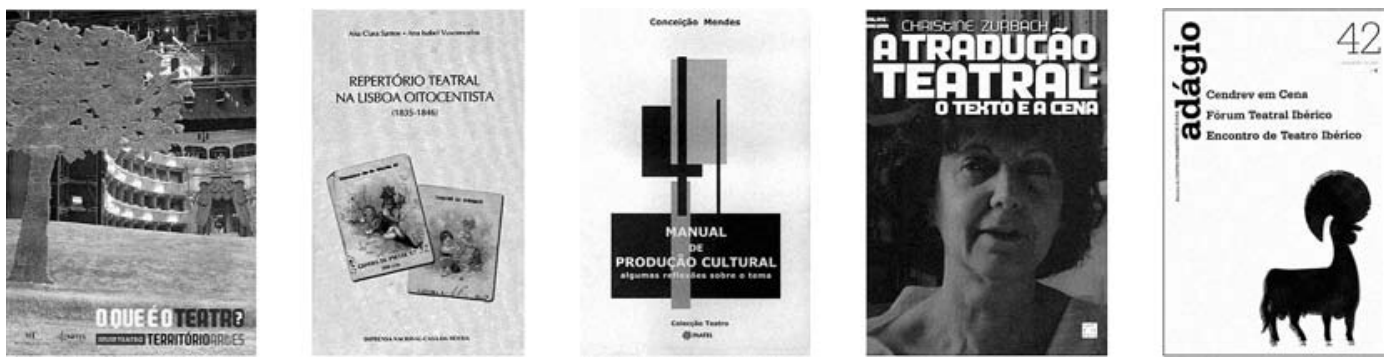

\section{Adenda à lista publicada na Sinais de cena n. $^{\circ} 7$}

ARTAUD, Antonin, 0 teatro e o seu duplo, trad. Fiama Hasse Brandão, pref. Vasco Tavares dos Santos, Lisboa, Fenda, 2006 [Estudos / Documentos].

Artistas Unidos: Revista, n.o 18 (Dezembro 2006), dir. Jorge Silva Melo, Lisboa, Livros Cotovia [Revista].

BARATA, José Oliveira / OLIVEIRA, Fernando Matos / SANTANA, Maria Helena (ed.), O melodrama, Coimbra, Centro de Literatura Portuguesa \&t Faculdade de Letras, 2006 [Estudos / Documentos].

BERGMAN, Ingmar, Fanny e Alexander, trad. Armando Silva Carvalho, Lisboa, Assírio \&t Alvim, 2006 [Peça].

CASTRO, Fernanda de, Teatro completo (A pedra no lago; A espada de cristal; Maria da Lua e Os cães não mordem), Lisboa, Círculo de Leitores, 2006 [Peças].

CABRAL, Carlos, Manual de encenação, Lisboa, INATEL, 2006 [Estudos / Documentos].

ESPADA, José, Manual de cenografia, Lisboa, INATEL, 2006 [Estudos / Documentos].

OLIVEIRA, Filomena / REAL, Miguel, 1755: 0 grande terramoto, pref. Carlos Fragateiro, Póvoa de Santo Adrião, Europress, 2006 [Peça].

PINHO, Sebastião Tavares de (coord.), Teatro neolatino em Portugal no contexto da Europa: 450 anos de Diogo de Teive, Coimbra, Imprensa da Universidade de Coimbra, 2006 [Estudos / Documentos].

RODRÍGUEZ, Gemma, Mal vistos, trad. Ana Vitorino, Carlos Costa e Catarina Martins, prefácio de Francisco Louçã, Vila Nova de Famalicão, Edições Quasi, 2006 [Peça].

SILVA, Maria de Fátima Sousa e, Furor: Ensaios sobre a obra dramática de Hélia Correia, Coimbra, Imprensa da Universidade de Coimbra, 2006 [Estudos / Documentos].

\section{Adenda à lista publicada na Sinais de cena $n .^{\circ} 3$}

CASTRO, Paulo, Red Shoes, pref. Jorge Reis-Sá, posfácio Paulo Brandão, Quasi Edições, Vila Nova de Famalicão, 2004 [Peça].

FERNANDES, Ângelo Miguel dos Passos, Albérico ponto final!, Lisboa, INATEL, Teatro, 2004 [Peça].

GERALDO, José, Inesperadamente na continuação ou Férias na barbearia com Elizabete, Lisboa, INATEL, 2004 [Peça].

SÉGUR, Condessa de, Os desastres de Sofia, com ilustrações de Maria Helena Vieira da Silva, Lisboa, Artemágica / Fundação Arpad Szenes-Vieira da Silva, 2004 [Peça].

\section{Adenda à lista publicada na Sinais de cena n. ${ }^{\circ} 1$}

MONTEIRO, Ofélia Paiva / SANTANA, Maria Helena (org.), Almeida Garrett: Um romântico, um moderno, Actas do Congresso Internacional Comemorativo do Bicentenário do Nascimento do Escritor, Lisboa, Imprensa Nacional-Casa da Moeda, Temas Portugueses, 2 vols., 2003 [Estudos / Documentos]. 